\title{
Articles
}

\section{Dissolution and Gelation of Cellulose in TBAF/DMSO Solutions: The Roles of Fluoride lons and Water}

\author{
Åsa Östlund, ${ }^{*, \dagger}$ Dan Lundberg, ${ }^{\ddagger}$ Lars Nordstierna, ${ }^{\dagger}$ Krister Holmberg, ${ }^{\dagger}$ and Magnus Nydén ${ }^{\dagger}$ \\ Department of Chemical and Biological Engineering, Applied Surface Chemistry, Chalmers University of \\ Technology, SE-412 96 Göteborg, Sweden, and Department of Chemistry, University of Coimbra, \\ 3004-535 Coimbra, Portugal
}

Received January 30, 2009; Revised Manuscript Received July 2, 2009

\begin{abstract}
Solutions of cellulose in a mixture of tetrabutylammonium fluoride and dimethyl sulfoxide (TBAF/DMSO) containing small and varying amounts of water were studied by nuclear magnetic resonance (NMR). By measuring the composition dependences of ${ }^{19} \mathrm{~F}$ NMR and ${ }^{1} \mathrm{H}$ NMR chemical shifts and line widths, details on the dissolution and gelation mechanisms for cellulose in TBAF/DMSO were elucidated. Our results suggest that the strongly electronegative fluoride ions act as hydrogen bond acceptors to cellulose hydroxyl groups, thus dissolving the polymer by breaking the cellulose-cellulose hydrogen bonds and by rendering the chains an effective negative charge. It was found that the fluoride ions also interact strongly with water. Small amounts of water remove the fluoride ions from the cellulose chains and allow reformation of the cellulose-cellulose hydrogen bonds, which leads to formation of highly viscous solutions or gels even at low cellulose concentrations.
\end{abstract}

\section{Introduction}

The current interest in biopolymers has triggered research to find new routes to dissolve cellulose, the most abundant polymer on earth. Native cellulose can have a molecular weight of up to $1.6 \times 10^{6} \mathrm{~mol} / \mathrm{g}$, which corresponds to a degree of polymerization (DP) of $1 \times 10^{4}$ glucose units. The attractive interaction between the polysaccharide chains is due to a combination of van der Waals forces and intermolecular hydrogen bonds. ${ }^{1}$ Both native and processed cellulose display amorphous as well as crystalline regions, where the latter are particularly difficult to dissolve. ${ }^{2}$ The key to get cellulose in solution is to overcome the strong intermolecular attractions between the chains.

In recent years, ionic liquids, such as salts of $N, N$-disubstituted imidazolines, have attracted considerable interest as solvents for cellulose. ${ }^{3}$ The combination of an aprotic, polar solvent, and an inorganic salt has also been found to have good solvency for cellulose, particularly if the salt contains a hard cation. Lithium chloride in dimethyl acetamide ( $\mathrm{LiCl} / \mathrm{DMAc})$ is one such system that was explored about 20 years ago and that has good dissolution power for cellulose. ${ }^{4} \mathrm{~A}$ drawback of this system is that the dissolution is time-consuming and requires preswelling by sequential solvent exchange and heating.

In year 2000, Heinze et al. reported that a solution of the trihydrate of tetrabutylammonium fluoride $\left(\mathrm{TBAF} \cdot 3 \mathrm{H}_{2} \mathrm{O}\right)$ in dimethyl sulfoxide (DMSO) is a good solvent for cellulose. ${ }^{5}$ This system can be used for rapid dissolution of celluloses of very high DP and is also capable of dissolving the complete plant cell wall. ${ }^{6} \mathrm{TBAF} / \mathrm{DMSO}$ has subsequently been explored as a reaction medium for chemical modifications of cellulose,

* To whom correspondence should be addressed. Tel.: +460317721000 . E-mail: asa.ostlund@chalmers.se.

Chalmers University of Technology.

University of Coimbra. for example, acetylation, ${ }^{5,7}$ carboxymethylation, ${ }^{8}$ and benzylation. ${ }^{9}$ However, the dissolution mechanism of this solvent system is not fully elucidated. DMSO, is a polar, aprotic solvent that can act as both a soft base (the sulfoxide sulfur) and a hard base (the sulfoxide oxygen). ${ }^{10}$ This dual functionality of DMSO makes it an excellent solvent for a wide range of substances, and although it cannot alone dissolve cellulose, it is one of the best swelling agents for the polymer. ${ }^{11,12}$ As to TBAF, it can be noted that the fluoride salt is the only halide salt of $\mathrm{TBA}^{+}$ capable of rendering cellulose soluble in DMSO; TBACl and $\mathrm{TBABr}$ are not useful in this respect. ${ }^{5}$ It has been suggested that the fluoride ion, which is a very strong hydrogen bond acceptor, favorably competes with the hydroxyl and acetal oxygen atoms of cellulose, ${ }^{7}$ thus reducing the attractive interactions between the polysaccharide chains. Chloride and bromide ions are evidently not efficient enough as hydrogen bond acceptors to do this.

The presence of water has a very strong influence on the physical properties of a solution of cellulose in TBAF/DMSO. Even a very small amount of water gives a strong increase in viscosity and a few percent of water causes gelation. Similar behavior has been observed in the $\mathrm{LiCl} / \mathrm{DMAc}$ system, and the influence of the water was attributed to competitive hydrogen bonding. ${ }^{13,14}$

The aim of the present paper is two-fold: to elucidate the mechanism of dissolution of cellulose in TBAF/DMSO and to explain the gelation that occurs on addition of water. The intermolecular interactions are monitored by ${ }^{19} \mathrm{~F}$ NMR and ${ }^{1} \mathrm{H}$ NMR. NMR experiments give information about the electronic environment experienced by a nucleus as well as about dynamic processes such as molecular mobility and chemical exchange, which are both important in the study of interactions between cellulose and solvents or cosolutes. ${ }^{15-17}$ It is a fortunate circumstance that one of the species of interest in the present 
Table 1. Compositions of the Investigated Samples, the ${ }^{19} \mathrm{~F}$ Chemical Shift of Fluoride Signal, $\delta_{(\mathrm{F}-)}$, the Half Height Peak Width of Fluoride, $\Delta v_{1 / 2(\mathrm{~F}-))}$, and the ${ }^{1} \mathrm{H}$ Chemical Shift of Water, $\delta_{\left(\mathrm{H}_{2} \mathrm{O}\right)}$

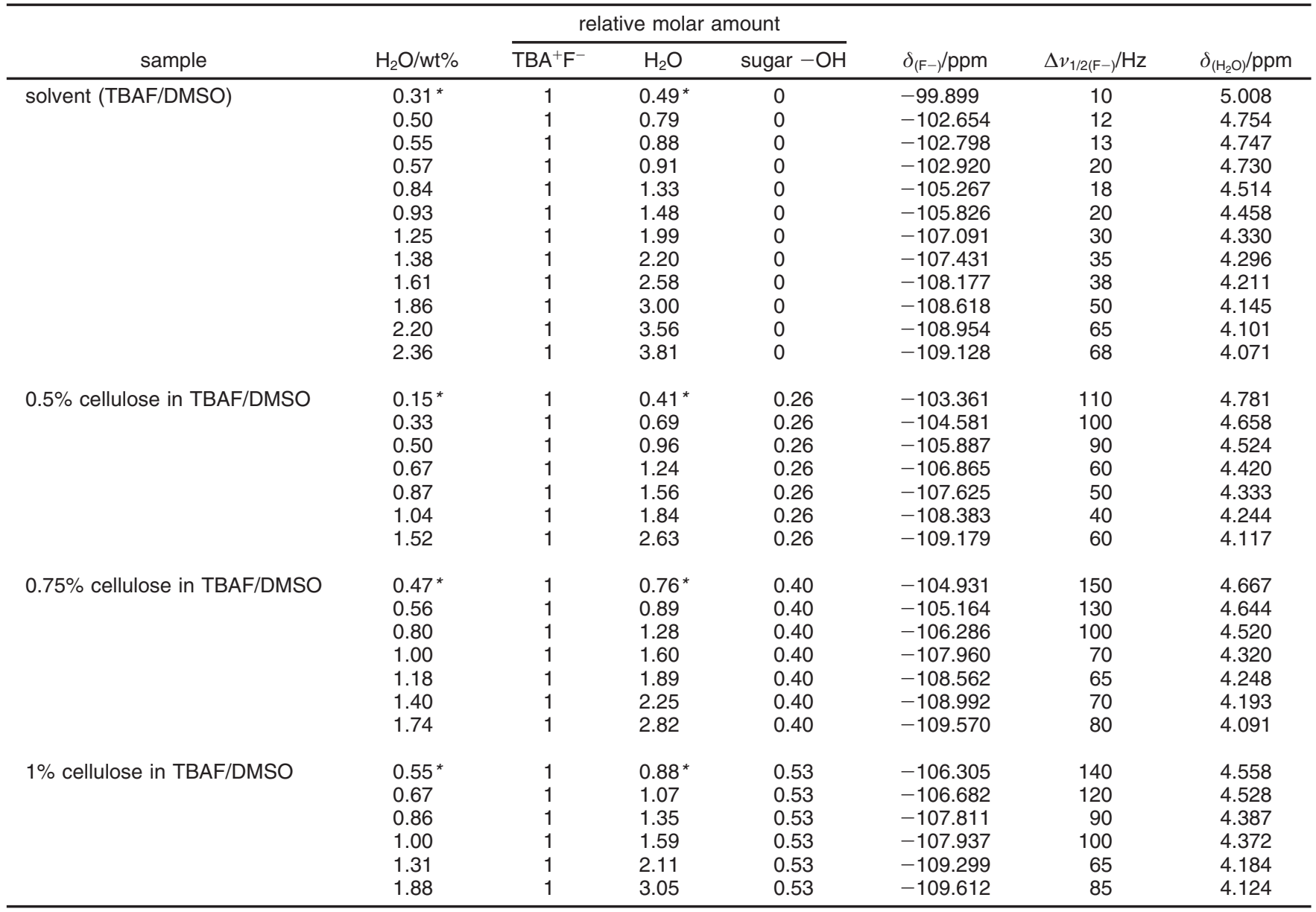

*The water content for these samples was achieved by extrapolation from the known water additions in each series.

investigation is the fluoride ion; the chemical shift of the peak from $\mathrm{F}^{-}$in ${ }^{19} \mathrm{~F}$ NMR is exceptionally sensitive to small changes in its chemical environment. ${ }^{18-22}$

\section{Experimental Section}

2.1. Materials. The cellulose used in all samples was Avicel $\mathrm{PH}$ 200 , a microcrystalline quality with a DP of $210-270,{ }^{23}$ which was purchased from FMC Europe. Tetrabutylammonium fluoride (TBAF), delivered as a $1 \mathrm{M}$ solution in tetrahydrofuran (THF), was obtained from Fluka, deuterated dimethyl sulfoxide (DMSO- $d_{6} ; 99.5$ atom $\%$ D) from ARMAR Chemicals, and $4 \AA$ molecular sieves from MERCK. The cellulose was dried at $120{ }^{\circ} \mathrm{C}$ for $24 \mathrm{~h}$ prior to use; otherwise, all chemicals were used as received. The water that was added to the samples was purified using Millipore Milli-Q equipment.

2.2. NMR Experiments. 2.2.1. Preparation of NMR Samples. The appropriate amounts of a $1 \mathrm{M}$ solution of TBAF in THF and DMSO$d_{6}$ were mixed to obtain a solution with a weight ratio of 1:11 of TBAF/ DMSO- $d_{6}$ (corresponding to $0.42 \mathrm{M}$ TBAF in DMSO), which is the same proportions as used by Heinze et al. ${ }^{5}$ This solution was then evaporated under vacuum $\left(60^{\circ} \mathrm{C}, 20 \mathrm{~min}\right)$ to decrease the amount of THF to below 1 wt $\%$. To further decrease the water content, the solution was stirred with activated molecular sieves for five days; the sieves were exchanged twice during this time. Dried cellulose was dissolved in the TBAF/DMSO solution during heating and stirring (60 ${ }^{\circ} \mathrm{C}, 20 \mathrm{~min}$ ) to give a cellulose concentration of $1.0 \mathrm{wt} \%$. This solution is clear to the eye and contains no visible undissolved particles. This $1 \%$ stock solution was then diluted with dried solvent to obtain the desired cellulose concentrations. Aliquots of the different cellulose solutions were transferred to $5 \mathrm{~mm}$ NMR tubes and the appropriate amounts of water were added. The samples were then vigorously vortexed with $3 \mathrm{~mm}$ glass beads (two in each sample). The tubes were flame-sealed to avoid uptake of additional water (the TBAF/DMSO mixture is inherently hygroscopic ${ }^{24}$ and centrifuged several times back and forth to mix and homogenize the samples. The samples were equilibrated for at least 1 week before characterization.

The lowest water concentration in each sample series was estimated by linear extrapolation of the ${ }^{1} \mathrm{H}$ NMR peak integrals from the five lowest water concentrations within the series to zero addition of water. For the sample series containing cellulose, the upper limit of the water content was restricted for practical reasons by high viscosity or gelation. The compositions of the investigated samples are presented in Table 1.

By using the above multistep procedure when preparing the TBAF/ DMSO solution, the problem of low solubility of the TBAF salt (which is only stable as a trihydrate, i.e. $\mathrm{TBAF} \cdot 3 \mathrm{H}_{2} \mathrm{O}$ ) in DMSO was circumvented. Complete drying of the TBAF $\cdot 3 \mathrm{H}_{2} \mathrm{O}$ is not possible since the salt decomposes at temperatures above $77^{\circ} \mathrm{C}^{7,25}$ and in the total absence of water. ${ }^{26}$

2.2.2. NMR Experimental Setup. All ${ }^{19} \mathrm{~F}$ NMR and ${ }^{1} \mathrm{H}$ NMR experiments, except reference experiments in the field dependency study (see below), were performed on a JEOL400 spectrometer operating at $376.5 \mathrm{MHz}$ for ${ }^{19} \mathrm{~F}$ and $400 \mathrm{MHz}$ for ${ }^{1} \mathrm{H}$, respectively. For each spectrum 32 scans were collected. The ${ }^{19} \mathrm{~F}$ NMR ppm scale was calibrated to an external sample containing trace amounts of fluorobenzene in TBAF/ DMSO; the chemical shift of the fluorobenzene peak was set to -113.1 ppm. ${ }^{27}$ Between experiments this peak shifted by less than $\pm 0.002 \mathrm{ppm}$, which verifies good stability of the magnetic field. In the ${ }^{1} \mathrm{H}$ NMR spectra the ppm scale was set after the DMSO signal at $2.5 \mathrm{ppm}$, which is independent of both $\mathrm{H}_{2} \mathrm{O}$ and cellulose content and hence did not 


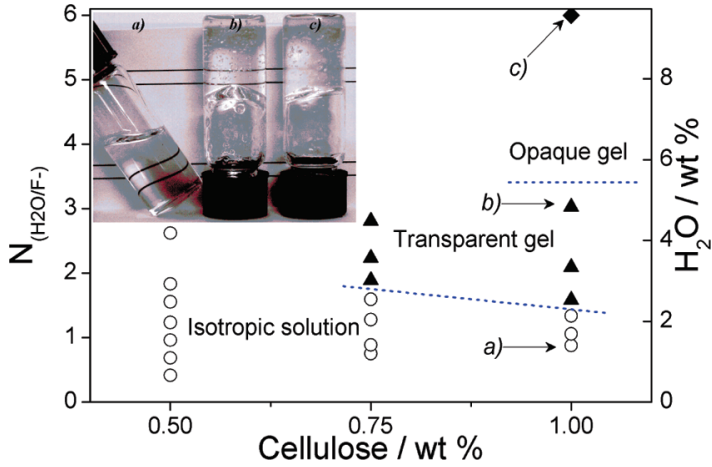

Figure 1. Photograph and plot of samples at varied cellulose and water content: $(\bigcirc)$ samples at isotropic solutions; $(\mathbf{\Delta})$ transparent gels; $(\diamond)$ opaque gels. Inspection of the parallel lines in the background of the photograph shows the difference in transparency between the samples. Vial (a) $1 \%$ cellulose (data point $a$ in plot); vial (b) $2.3 \%$ $\mathrm{H}_{2} \mathrm{O}$ and $1 \%$ cellulose (data point $b$ in plot); vial (c) $10 \% \mathrm{H}_{2} \mathrm{O}$ and $1 \%$ cellulose (data point $c$ in plot) all in TBAF/DMSO.

shift between samples. Unless otherwise stated, all NMR experiments were performed at $22( \pm 0.1){ }^{\circ} \mathrm{C}$.

The dependence of the ${ }^{19} \mathrm{~F}$ line width on the magnetic field strength was investigated by comparing the spectra obtained at $376.5 \mathrm{MHz}$ to spectra obtained on a Varian INOVA spectrometer working at 565.9 $\mathrm{MHz}$ at otherwise identical conditions.

The temperature dependence studies were performed by recording spectra at $22,30,40$, and $50( \pm 0.1){ }^{\circ} \mathrm{C}$. At each temperature both ${ }^{19} \mathrm{~F}$ NMR and ${ }^{1} \mathrm{H}$ NMR spectra were collected. After each temperature change the samples were conditioned for at least $30 \mathrm{~min}$ before the experiments to allow equilibration.

\section{Results}

3.1. Visual Appearance. As illustrated in Figure 1, samples within the investigated composition ranges, that is, up to $1 \mathrm{wt}$ $\%$ cellulose and molar ratios of $\mathrm{H}_{2} \mathrm{O}$ to $\mathrm{F}^{-}\left(\mathrm{N}_{\left(\mathrm{H}_{2} \mathrm{O} / \mathrm{F}-\right)}\right)$ up to 6 , can be classified with respect to their physical appearance into three categories: isotropic solutions, transparent gels, and opaque gels. At low concentrations of both cellulose and water, samples are clear isotropic solutions, see vial $a$ in Figure 1. The viscosity of the solutions increases with increasing concentration of either solute. At a certain solute concentration the solutions turn into rigid transparent gels as illustrated by vial $b$, which is turned upside-down in Figure 1. At high water concentration the gels gradually become turbid as exemplified by vial $c$ in Figure 1. Samples classified as opaque gels (such as that in vial $c$ ) could not be studied by NMR, due to the high degree of phase separation which causes heterogeneities in the sample.

3.2. ${ }^{19}$ F NMR. 3.2.1. Composition Dependence. The dependence of $\mathrm{N}_{\left(\mathrm{H}_{2} \mathrm{O} / \mathrm{F}-\right)}$ on the chemical shift, $\delta_{(\mathrm{F}-)}$, and the half height peak width, $\Delta v_{1 / 2(\mathrm{~F}-)}$, for the fluoride signals is shown in Figure 2. As can be seen in Figure 2a, an increase in $\mathrm{N}_{\left(\mathrm{H}_{2} \mathrm{O} / \mathrm{F}-\right)}$ results in a significant decrease of the fluoride ion chemical shift $\left(\delta_{(\mathrm{F}-)}\right)$, in all sample series, that is, both with and without cellulose. Addition of $0.5 \%$ cellulose or more to the pure TBAF/DMSO solution causes a small but significant decrease in $\delta_{(\mathrm{F}-)}$, for all $\mathrm{N}_{\left(\mathrm{H}_{2} \mathrm{O} / \mathrm{F}-\right)}$. It is interesting to find, however, that an increase in the cellulose concentration from 0.5 to $1 \%$ causes no notable change in $\delta_{(\mathrm{F}-)}$. For all sample series, $\delta_{(\mathrm{F}-)}$ starts to level out to a value close to $-110 \mathrm{ppm}$ when $\mathrm{N}_{\left(\mathrm{H}_{2} \mathrm{O} / \mathrm{F}-\right)}$ is higher than 2 (Figure 2a).

It can be seen in Figure $2 \mathrm{~b}$ that $\Delta v_{1 / 2(\mathrm{~F}-)}$ is strongly dependent on $\mathrm{N}_{\left(\mathrm{H}_{2} \mathrm{O} / \mathrm{F}-\right)}$. In the pure TBAF/DMSO there is a monotonic increase in $\Delta v_{1 / 2(\mathrm{~F}-)}$ from 10 to $68 \mathrm{~Hz}$ when $\mathrm{N}_{\left(\mathrm{H}_{2} \mathrm{O} / \mathrm{F}-\right)}$ increases
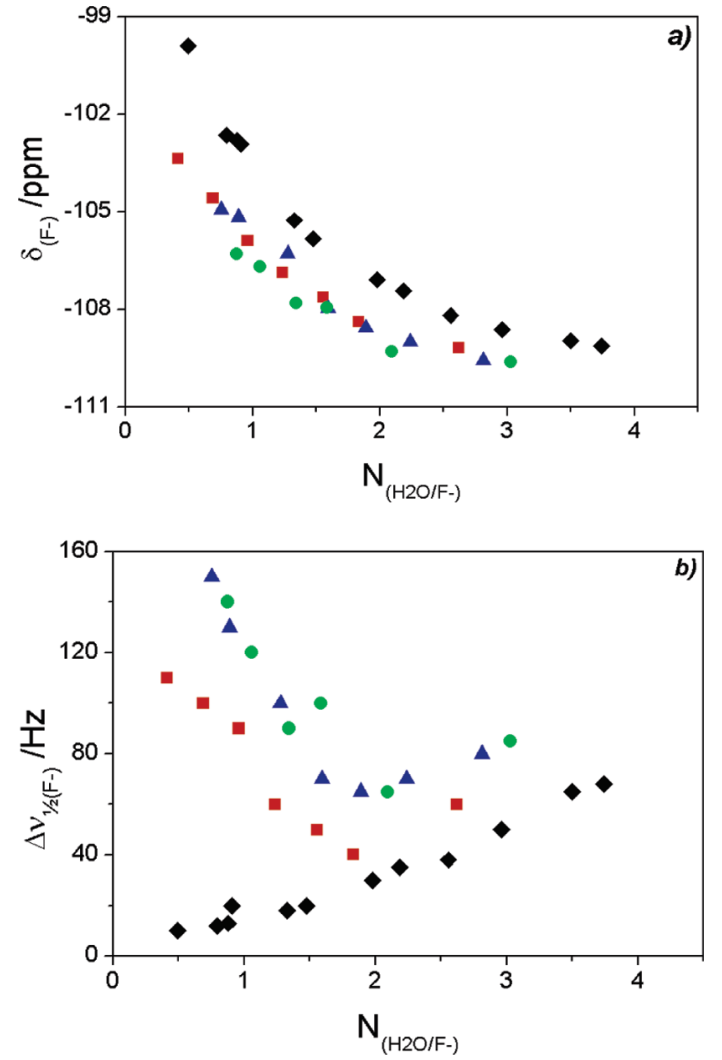

Figure 2. Dependence of ${ }^{19} \mathrm{~F}$ NMR signals from fluoride vs the ratio

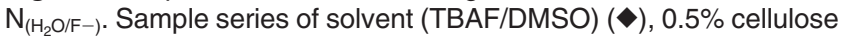
(red square), $0.75 \%$ cellulose (blue triange), and $1 \%$ cellulose (green circle), at 376.5 MHz. (a) $\mathrm{F}^{-}$shift, $\delta_{(\mathrm{F}-)}$, dependence on $\mathrm{N}_{\left(\mathrm{H}_{2} \mathrm{O} / \mathrm{F}-\right)}$; (b) $\mathrm{F}^{-}$line-broadening, $\Delta v_{1 / 2(\mathrm{~F}-)}$, dependence on $\mathrm{N}_{\left(\mathrm{H}_{2} \mathrm{O} / \mathrm{F}-\right)}$.

from 0.49 to 3.81 (which corresponds to an increase in the water content from 0.31 to $2.36 \mathrm{wt} \%$ ). With cellulose present in the system, $\Delta v_{1 / 2(\mathrm{~F}-)}$ consistently shows a nonmonotonic dependence of $\mathrm{N}_{\left(\mathrm{H}_{2} \mathrm{O} / \mathrm{F}^{-}\right)}$and there is a minimum in $\Delta v_{1 / 2(\mathrm{~F}-)}$ close to $\mathrm{N}_{\left(\mathrm{H}_{2} \mathrm{O} /\right.}$ $\mathrm{F}-)=2$ for all three studied cellulose concentrations. For the lowest water contents, $\Delta v_{1 / 2(\mathrm{~F}-)}$ increases from $10 \mathrm{~Hz}$ in pure TBAF/DMSO to $100-150 \mathrm{~Hz}$ with cellulose present. At the minimum $\Delta v_{1 / 2}(\mathrm{~F}-)$ in the series with $0.5 \%$ cellulose is similar to $\Delta v_{1 / 2(\mathrm{~F}-)}$ for pure TBAF/DMSO, that is, $40 \mathrm{~Hz}$, whereas the samples containing 0.75 and $1 \%$ cellulose both show $\Delta v_{1 / 2(\mathrm{~F}-)}$ of $65 \mathrm{~Hz}$. With $\mathrm{N}_{\left(\mathrm{H}_{2} \mathrm{O} / \mathrm{F}-\right)}$ above 2, that is, at the same value as $\delta_{(\mathrm{F}-)}$, starts to level out, $\Delta v_{1 / 2(\mathrm{~F}-)}$ changes roughly parallel to the values in TBAF/DMSO for all cellulose contents.

3.2.2. Magnetic Field Dependence. To identify a possible field dependence of the line width of the fluoride signal in solutions with or without cellulose, ${ }^{19} \mathrm{~F}$ NMR spectra of samples containing pure solvent or $0.5 \%$ cellulose with a varying $\mathrm{N}_{\left(\mathrm{H}_{2} \mathrm{O} / \mathrm{F}-\right)}$ were recorded at two different field strengths. The results are shown in Figure 3. For the pure TBAF/DMSO series, $\Delta v_{1 / 2\left(\mathrm{~F}^{-}\right)}$shows no appreciable field dependence. When cellulose is present, however, the values of $\Delta v_{1 / 2\left(\mathrm{~F}^{-}\right)}$are consistently larger at higher field for the whole presented range of $\mathrm{N}_{\left(\mathrm{H}_{2} \mathrm{O} / \mathrm{F}-\right)}$, with the difference being more pronounced at low $\mathrm{N}_{\left(\mathrm{H}_{2} \mathrm{O} / \mathrm{F}-\right)}$.

3.2.3. Temperature Dependence. The temperature dependences of the shift and width of the fluoride signal in samples containing $1 \%$ cellulose but different amounts of water, corresponding to $\mathrm{N}_{\left(\mathrm{H}_{2} \mathrm{O} / \mathrm{F}-\right)}$ of $0.88,1.35$, and 3.05 , are shown in Figure 4. Also shown are results in pure TBAF/DMSO solutions with $\mathrm{N}_{\left(\mathrm{H}_{2} \mathrm{O} / \mathrm{F}-\right)}$ of 0.49 and 2.58 . Both $\delta_{(\mathrm{F}-)}$ and $\Delta v_{1 / 2(\mathrm{~F}-)}$ show notable temperature dependence. There is a similar degree of decrease in chemical shift with increasing temperature for all samples. 


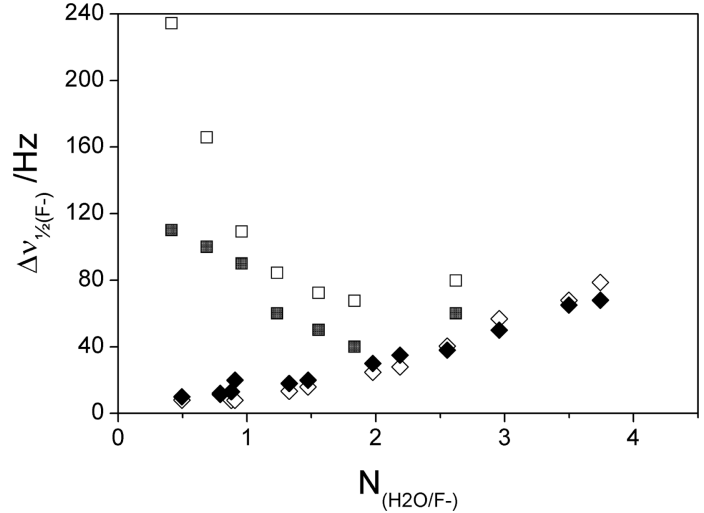

Figure 3. Dependence of the fluoride signal line-width, $\Delta v_{1 / 2(\mathrm{~F}-)}$, on $\mathrm{N}_{\left(\mathrm{H}_{2} \mathrm{O} / \mathrm{F}-\right)}$ and on magnetic field strength. Experiments at $376.5 \mathrm{MHz}$ are represented with filled symbols,: TBAF/DMSO ( $)$ and $0.5 \%$ cellulose (ם). Experiments at $565.9 \mathrm{MHz}$ are represented with unfilled symbols: TBAF/DMSO $(\diamond)$ and $0.5 \%$ cellulose $(\square)$.

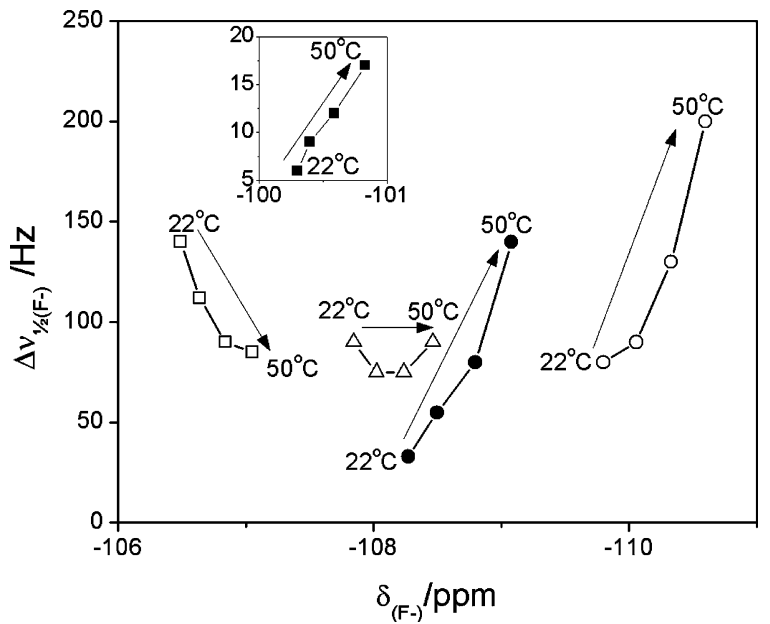

Figure 4. Temperature dependence of the line width, $\Delta v_{1 / 2(\mathrm{~F}-)}$, and the chemical shift of the fluoride signal, $\delta_{(\mathrm{F}-)}$, of samples with varying $\mathrm{N}_{\left(\mathrm{H}_{2} \mathrm{O} / \mathrm{F}-\right)}$ and in presence or absence of cellulose. Filled symbols represent pure TBAF/DMSO solution at $\mathrm{N}_{\left(\mathrm{H}_{2} \mathrm{O} / \mathrm{F}-\right)}$ of 0.49 (घ), and 2.58 $(\bullet)$; whereas unfilled symbols represents samples in the presence of cellulose at $\mathrm{N}_{\left(\mathrm{H}_{2} \mathrm{O} / \mathrm{F}-\right)}$ of 0.88 with $1 \%$ cellulose ( $\left.\square\right), 1.35$ with $1 \%$ cellulose $(\Delta)$, and 3.05 with $1 \%$ cellulose $(\bigcirc)$. Experiments were run at $22,30,40$, and $50^{\circ} \mathrm{C}$. The effect of an increased temperature is clarified by the directions of the arrows.

However, in the case of $\Delta v_{1 / 2(\mathrm{~F}-)}$, opposite trends are observed for low and high water concentrations in the presence of cellulose. With $\mathrm{N}_{\left(\mathrm{H}_{2} \mathrm{O} / \mathrm{F}-\right)}=0.88$ the $\Delta v_{1 / 2}(\mathrm{~F}-)$ decreases with temperature, whereas with $\mathrm{N}_{\left(\mathrm{H}_{2} \mathrm{O} / \mathrm{F}-\right)}=3.05$ it increases. For the intermediate water concentration, corresponding to $\mathrm{N}_{\left(\mathrm{H}_{2} \mathrm{O} / \mathrm{F}-\right)}=$ 1.35, a minimum in $\Delta v_{1 / 2}(\mathrm{~F}-)$ is observed. In the absence of cellulose, $\Delta v_{1 / 2}(\mathrm{~F}-)$ increases with temperature for both studied water concentrations.

3.2.4. Additional ${ }^{19} F$ NMR Signals. In addition to the peak from the fluoride ion, signals were found at $-123,-143$, and $-148 \mathrm{ppm}$ (for ${ }^{19} \mathrm{~F}$ NMR spectra see Supporting Information). The signal at $-123 \mathrm{ppm}$ most likely originates from $\mathrm{SiF}_{6}{ }^{2-}$ generated from HF attacking the glass sample tube, ${ }^{28}$ and the two latter signals probably correspond to activities of $\mathrm{HF}_{2}{ }^{-}$in equilibrium with $\mathrm{HF}^{29}$ However, these three signals have intensities of the order of $100 \times$ lower than the fluoride signal and are of no relevance for the present study.

3.3. ${ }^{1}$ H NMR. 3.3.1. Composition Dependence. Figure 5a,b shows ${ }^{1} \mathrm{H}$ NMR spectra with assignments of samples with pure TBAF/DMSO or $1 \%$ of cellulose at the same content of water.
A comparison of the ${ }^{1} \mathrm{H}$ NMR spectra for different samples shows that the shift of the DMSO peak is practically independent of sample composition $\left(\delta_{(\mathrm{DMSO})}=2.504 \pm 0.001 \mathrm{ppm}\right.$ in all investigated samples). The $\mathrm{TBA}^{+}$signals show only minor changes in shift with varying composition; the signal from the $\alpha$-protons shift decrease by $0.045 \mathrm{ppm}$ when the water content is increased from 0.31 to $2.36 \%$ and a shift decrease by 0.010 ppm when $1 \%$ of cellulose is added to the pure TBAF/DMSO, whereas the other $\mathrm{TBA}^{+}$signals are practically independent of composition.

Figure 6 shows the dependence of $\delta_{(\mathrm{H} 2 \mathrm{O})}$ and $\Delta v_{1 / 2(\mathrm{H} 2 \mathrm{O})}$ on $\mathrm{N}_{\text {(H2O/F-) }}$ for the water signal. The decrease in the chemical shift of the water signal seen in Figure 6a, is a consequence of a significantly increased shielding with increasing water content. At high $\mathrm{N}_{(\mathrm{H} 2 \mathrm{O} / \mathrm{F}-)}$ the shift starts to level out and approaches the shift of water in pure DMSO, i.e. $3.3 \mathrm{ppm}$. In the presence of cellulose the dependence of $\delta_{\left(\mathrm{H}_{2} \mathrm{O}\right)}$ on $\mathrm{N}_{\left(\mathrm{H}_{2} \mathrm{O} / \mathrm{F}-\right)}$ essentially parallels the trend observed in the pure TBAF/DMSO, but with the values of $\delta_{\left(\mathrm{H}_{2} \mathrm{O}\right)}$ consistently somewhat lower. Figure $6 \mathrm{~b}$ shows that the presence of cellulose causes broadening of the water peak and that the line width is only slightly dependent on the water concentration.

3.3.2. Temperature Dependence of the Water Signal. In Figure 7 the temperature dependence of the line width of the water signal is displayed for four comparable samples with or without cellulose and at two different water contents. For all samples, the water peak shows similar dependence of temperature, with respect to both shift change and line broadening, independent of the presence of cellulose.

\section{Discussion}

The results obtained in this study have highlighted the interactions of importance for dissolution and gelation of cellulose in TBAF/DMSO. There are many possible interactions between the components $\mathrm{H}_{2} \mathrm{O}$, DMSO, $\mathrm{TBA}^{+}, \mathrm{F}^{-}$, and cellulose, which may potentially play important roles for the behavior of the system. By varying the concentrations of water and cellulose, the influence of these components in the studied solutions were monitored by ${ }^{19} \mathrm{~F}$ NMR and ${ }^{1} \mathrm{H}$ NMR. The results strongly suggest that the most important interactions are those between fluoride ions, water, and cellulose. In the following discussion, these are, thus, the components in focus.

The strong effect of water on the macroscopic behavior of the samples is visualized by the samples shown in Figure 1. A gradual addition of water to a solution of cellulose in TBAF/ DMSO first causes an increase in the viscosity, then induces gelation, and at $\mathrm{N}_{\left(\mathrm{H}_{2} \mathrm{O} / \mathrm{F}-\right)}$ above about 3.5 renders the gel an opaque appearance. The induced and increasing turbidity can be ascribed to phase separation and to an increase in the number and size of the cellulose aggregates.

In the NMR experiments the influence of water on a molecular level is manifested by a strong dependence on $\mathrm{N}_{\left(\mathrm{H}_{2} \mathrm{O} / \mathrm{F}-\right)}$ of the water signal, as observed by ${ }^{1} \mathrm{H}$ NMR (Figure 6), as well as of the fluoride signal as observed by ${ }^{19} \mathrm{~F}$ NMR (Figure 2). We will start by discussing the fluoride-water interactions on the basis of the chemical shift dependences of the $\mathrm{F}^{-}$and water signals in pure TBAF/DMSO. In Figure $2 \mathrm{a}$, one can note that $\delta_{(\mathrm{F}-)}$ decreases with increasing $\mathrm{N}_{\left(\mathrm{H}_{2} \mathrm{O} / \mathrm{F}-\right)}$, which indicates a gradually increasing shielding of the fluoride ion. This shielding can probably be attributed to the change in electronic surrounding as the $\mathrm{F}^{-}$goes from a DMSO rich to a more water rich environment. The change in shielding can be attributed to a change in the average binding length. $\mathrm{F}^{-} \cdot \cdots \mathrm{H}$ binding lengths 


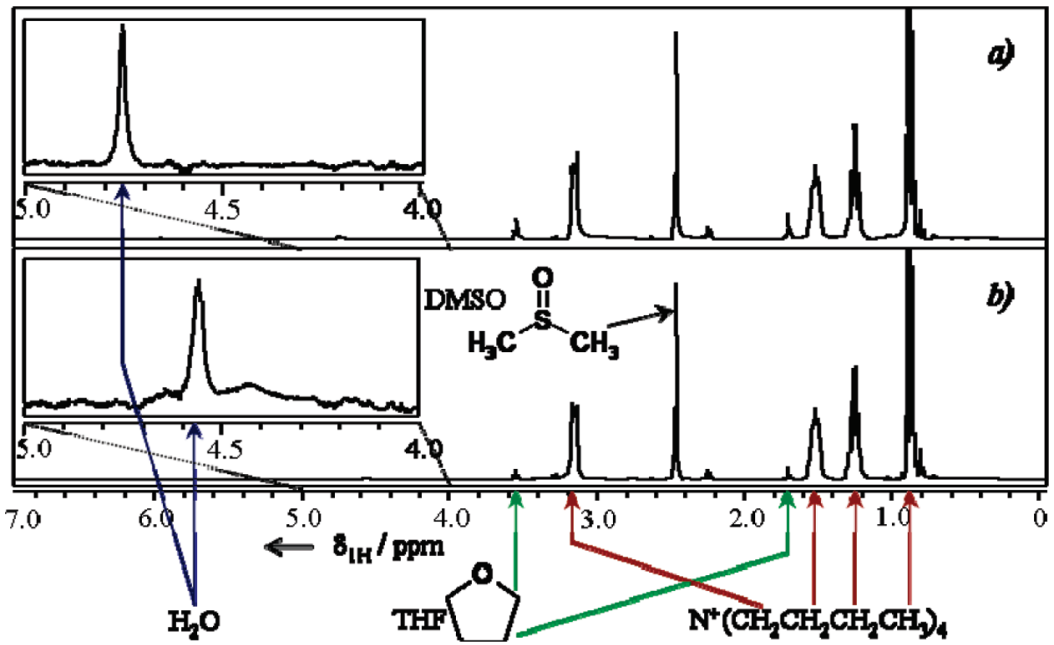

Figure 5. ${ }^{1} \mathrm{H}$ NMR with assignments of peaks from TBA ${ }^{+}$, THF, DMSO, and $\mathrm{H}_{2} \mathrm{O}$ : (a) $0.55 \% \mathrm{H}_{2} \mathrm{O}$ in TBAF/DMSO; (b) $0.55 \% \mathrm{H}_{2} \mathrm{O}$ in $1 \%$ cellulose (TBAF/DMSO).
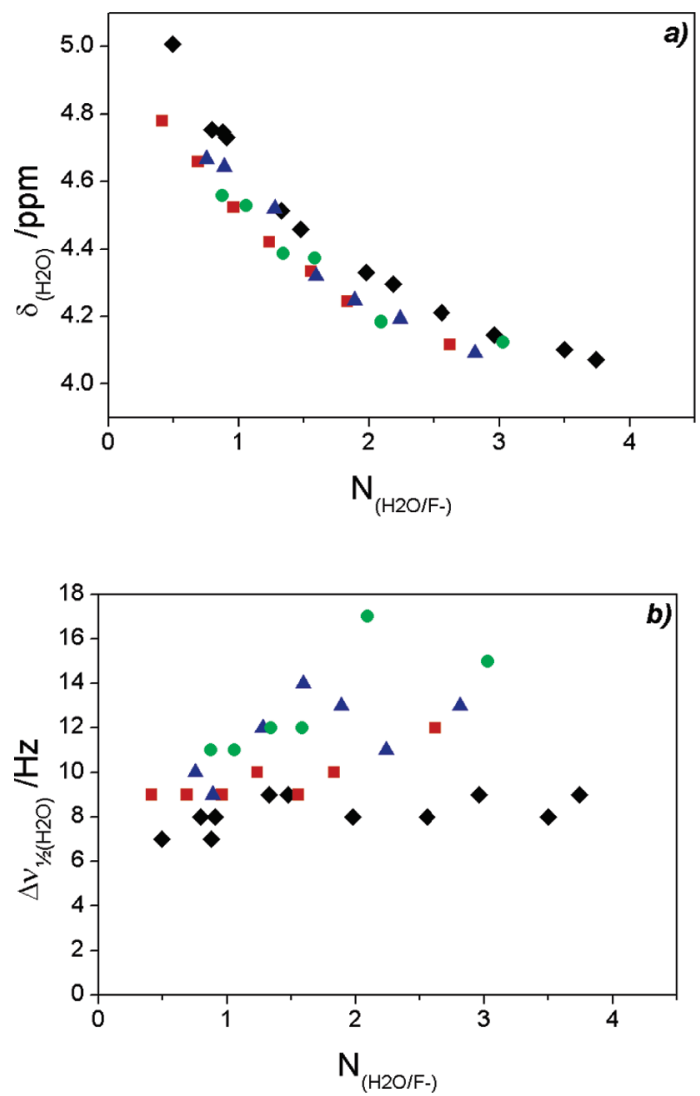

Figure 6. ${ }^{1} \mathrm{H}$ NMR of the $\mathrm{H}_{2} \mathrm{O}$ signal vs the ratio $\mathrm{N}_{\left(\mathrm{H}_{2} \mathrm{O} / \mathrm{F}-\right)}$. Sample series of solvent (TBAF/DMSO) $(\downarrow), 0.5 \%$ cellulose (red square), $0.75 \%$ cellulose (blue triangle), and $1 \%$ cellulose (green circle), at 376.5 MHz. (a) Dependence of $\mathrm{N}_{\left(\mathrm{H}_{2} \mathrm{O} / \mathrm{F}-\right)}$ on $\mathrm{H}_{2} \mathrm{O}$ shift, $\delta_{\left(\mathrm{H}_{2} \mathrm{O}\right)}$. (b) Dependence of $\mathrm{N}_{\left(\mathrm{H}_{2} \mathrm{O} / \mathrm{F}-\right)}$ on $\mathrm{H}_{2} \mathrm{O}$ half peak height line width, $\Delta v_{1 / 2\left(\mathrm{H}_{2} \mathrm{O}\right)}$.

(where $\mathrm{H}$ derives from the solvent) in DMSO and water have been estimated to 1.876 and $1.415 \AA$, respectively. ${ }^{30} \mathrm{By}$ comparing the trend in $\delta_{(\mathrm{F}-)}$ with increasing water content to the values in the pure solvents, that is, $-73 \mathrm{ppm}$ in DMSO and $-119 \mathrm{ppm}$ in water, ${ }^{31}$ one can indeed see that the local environment of the fluoride ion becomes significantly more aqueous-like. It is interesting to find that the functional form of the chemical shift dependence of the water signal, as observed by ${ }^{1} \mathrm{H}$ NMR (Figure 6a), closely resembles that of the fluoride signal in ${ }^{19} \mathrm{~F}$ NMR. This suggests that the changes in electronic/

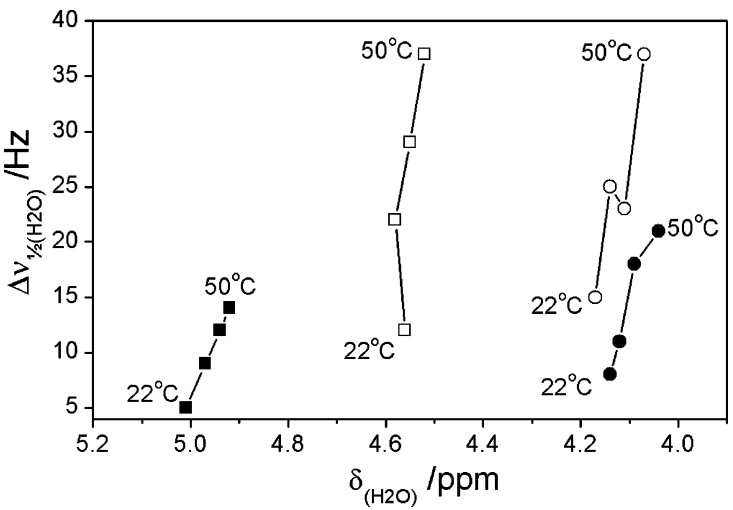

Figure 7. Temperature dependence of the line broadening, $\Delta v_{1 / 2\left(\mathrm{H}_{2} \mathrm{O}\right)}$, and the chemical shift of the water signal, $\delta_{\left(\mathrm{H}_{2} \mathrm{O}\right)}$, for samples with varying $\mathrm{N}_{\left(\mathrm{H}_{2} \mathrm{O} / \mathrm{F}-\right)}$ and in the presence or absence of cellulose. Filled symbols represent TBAF/DMSO solution at $\mathrm{N}_{\left(\mathrm{H}_{2} \mathrm{O} / \mathrm{F}-\right)}$ of 0.49 ( $\left.\square\right)$ and $2.58(0)$; and unfilled symbols represent samples in the presence of cellulose as $\mathrm{N}_{\left(\mathrm{H}_{2} \mathrm{O} / \mathrm{F}-\right)}$ of 0.88 with $1 \%$ cellulose $(\square)$ and 3.05 with $1 \%$ cellulose $(O)$. Experiments were run at $22,30,40$, and $50^{\circ} \mathrm{C}$.

chemical surrounding with $\mathrm{N}_{\left(\mathrm{H}_{2} \mathrm{O} / \mathrm{F}-\right)}$ for these two species are coupled, which supports the existence of significant fluoridewater interactions, as an increase in fluoride ions are transferred from its DMSO surrounding to the vicinity of $\mathrm{H}_{2} \mathrm{O}$ and thereby increases the electron density in the closest surrounding of $\mathrm{H}_{2} \mathrm{O}$.

One can find important clues about interactions between the different components in a solution not only by investigating the interdependence on the changes in chemical shifts, but also from changes in the appearance of the NMR signals. In general, line-broadening can arise from either chemical shift dispersion due to slow chemical exchange, an effect which usually shows a strong dependence on magnetic field strength, $B_{0}$, or from an increase in spin-spin relaxation rate, which on the other hand is relatively independent of $B_{0}$. Thus, by assessing the dependence of the peak shape on magnetic field strength it is possible to obtain information about the underlying mechanism of the broadening. As described by Furó et al., ${ }^{20}$ the width of the individual spectral lines scales approximately linearly with the applied magnetic field.

It can be seen in Figure $2 b$ that the line width of the fluoride signal in pure solvent increases with increasing $\mathrm{N}_{\left(\mathrm{H}_{2} \mathrm{O} / \mathrm{F}-\right)}$. From the results shown in Figure 3 one can conclude that the linebroadening observed in this case shows no field dependency and that its origin thus lies primarily in an increased spin-spin 


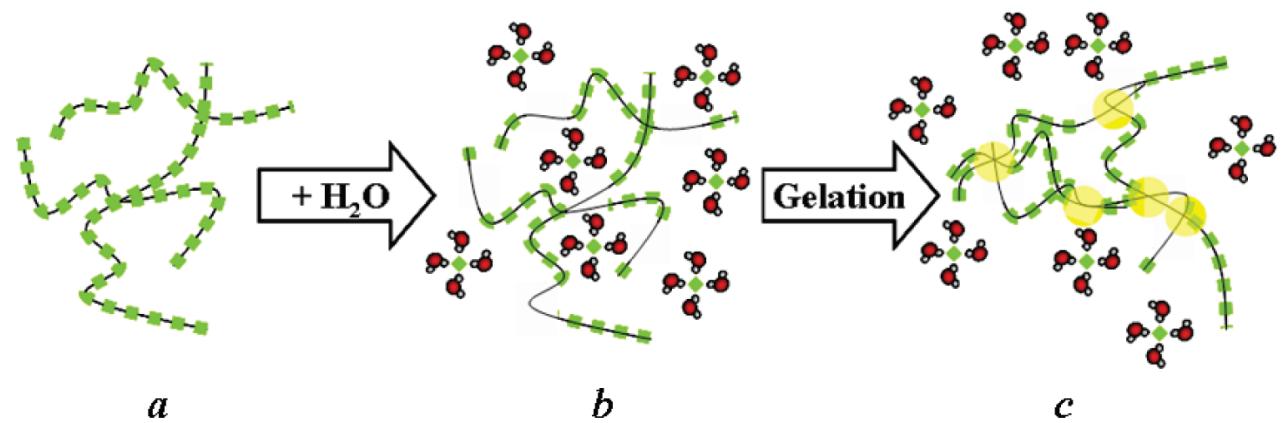

Figure 8. Schematic illustration of the mechanism for gelation of cellulose dissolved in TBAF/DMSO, where green squares represents $\mathrm{F}^{-}$, black lines represent the cellulose chains, and yellow circles highlight the hydrogen bonds: (a) cellulose in solution; (b) added water associates with $\mathrm{F}^{-}$ions and withdraws $\mathrm{F}^{-}$from cellulose; (c) parts of the cellulose chains will be exposed, which makes reaggregation possible.

relaxation rate with increasing water content. This finding is reasonable because the number of water molecules coordinated to $\mathrm{F}^{-}$increases with an increased amount of available water, and an increase in the effective size of an entity in solution gives a decrease in the rotation rate and, thus, an increased relaxation rate.

The situation gets more complex when cellulose is added to the system, and even $0.5 \%$ cellulose gives a significant decrease in $\delta_{(\mathrm{F}-)}$, in comparison to the value in pure solvent (Figure 2a). This finding is consistent with the idea that fluoride ions interact not only with the water molecules but also with the hydroxyl groups on the cellulose chain. The notable minimum in $\Delta v_{1 / 2}$ (around $\mathrm{N}_{\left(\mathrm{H}_{2} \mathrm{O} / \mathrm{F}-\right)}=2$ ) that is observed in the presence of cellulose (Figure 2b) is consistent with a changeover in the dominant "binding site" for $\mathrm{F}^{-}$. The broad signal at low water concentrations can be explained by a major fraction of the fluoride ions being bound to the large, inflexible cellulose, whereas the initial decrease in line-width with increasing water concentration can be attributed to dissociation of $\mathrm{F}^{-}$from the cellulose and inclusion of the ions in water clusters. This mechanism is further supported by the fact that for $\mathrm{N}_{\left(\mathrm{H}_{2} \mathrm{O} / \mathrm{F}-\right)}$ above 2 , the change in $\Delta v_{1 / 2}$ closely parallels the behavior in the pure solvent. As shown in Figure 3, the sample containing cellulose shows notable field dependence at low water contents. This observation suggests that chemical shift dispersion contributes to the line broadening, which can be explained by the $\mathrm{F}^{-}$being bound to different sites on the cellulose (each giving rise to a different $\left.\delta_{(\mathrm{F}-)}\right)$ in combination with a rather slow exchange between these sites.

The results from the temperature dependence studies give further support for the mechanism suggested above. A plausible explanation for the decrease in $\Delta v_{1 / 2}$ for $\mathrm{F}^{-}$with increasing temperature at low water content and in the presence of cellulose, as shown in Figure 4, is an increased rate of exchange of $\mathrm{F}^{-}$between the different association sites on the cellulose. At higher water content the change in $\Delta v_{1 / 2}$ with temperature is very similar with and without cellulose, which indicates that the signals predominantly originate from molecules in the bulk.

For the signals from $\mathrm{F}^{-}$, as well as those from $\mathrm{H}_{2} \mathrm{O}$, the chemical shift decreases with increasing temperature (Figures 4 and 7), a finding that gives further support for strong interactions between these species. These enhanced intermolecular interactions between $\mathrm{F}^{-}$and $\mathrm{H}_{2} \mathrm{O}$ also result in increased dipolar interactions and thereby an increase in line width, $\Delta v_{1 / 2}$, for both $\mathrm{F}^{-}$and $\mathrm{H}_{2} \mathrm{O}$ signals (Figures 4 and 7).

The absence of shift changes and line broadening for the $\mathrm{TBA}^{+}$signals on addition of cellulose suggest that this ion does not interact strongly with the polymer in the investigated composition range. In one previous report it has been proposed that $\mathrm{TBA}^{+}$might act as a swelling agent by adsorbing to the cellulose $^{32}$ and another paper emphasizes the benefits of using a bulky cation (known to be beneficial for ionic liquids). ${ }^{33}$ Whereas the first explanation is not supported by the present data, the latter is consistent with the findings; the use of a bulky counterion to $\mathrm{F}^{-}$allows for a better dissociation of the $\mathrm{F}^{-}$from the ion pair. ${ }^{34}$ This is why sodium fluoride in DMSO at equivalent concentration does not dissolve cellulose. Another study shows that TBA+ can be replaced by benzyltrimethylammonium fluoride (BTMAF) in DMSO with retention of the capability to dissolve cellulose. ${ }^{35}$ We believe that the most important reason for why a bulky cation to $\mathrm{F}^{-}$is necessary is its ability to enhance dissociation of the salt, while the capability of the cation to interact with cellulose through dispersion forces has less impact.

Finally, we would like to make some general comments on designing a solvent system for cellulose. First of all, a highly electronegative ion with strong hydrogen acceptor ability should be used to break the strong hydrogen bonds between the cellulose chains. The power of $\mathrm{F}^{-}$as a hydrogen bond acceptor is well-known and has previously been discussed in comparison to other halides in the context of solvents for cellulose. ${ }^{5} \mathrm{~A}$ parallel can be made to studies of the efficiency of ionic liquids to dissolve macromolecules, which have shown that the more electronegative the anion, the bulkier the cation should be. . $^{36-38}$ It can also be concluded that a small cation demands a bulky and highly polarizable anion in accordance with the Hofmeister series. ${ }^{39}$ Taken together, the salt should be properly dissociated in the bulk solvent (DMSO in this case) and either the anion or the cation needs to have a good hydrogen bonding ability.

\section{Concluding Remarks}

Our results suggest that the dissolution of cellulose in TBAF/ DMSO is primarily due to strong ion-dipole interactions between the fluoride ions from TBAF and the hydroxyl groups on cellulose, where the high ability of fluoride as a hydrogen bond acceptor effectively breaks the network of hydrogen bonds between the cellulose chains.

$\mathrm{F}^{-}$interacts strongly with both cellulose and water and the dissolving power of TBAF/DMSO can be eliminated in the presence of water. Gelation occurs when the electrostatic stabilization of the individual cellulose chains decreases as fluoride ions desorb from the polymer and instead coordinate with water in the bulk solvent. The suggested mechanism of the gelation is summarized schematically in Figure 8. With no, or little, water the cellulose is protected from self-association by the adsorbed fluoride ions (picture $a$ ). On addition of water, the fluoride ions are withdrawn from the cellulose chains into 
the bulk solvent (picture $b$ ). This causes the cellulose to interact via hydrogen bonds and a cross-linked gel is formed (picture c). To avoid gelation and, thus, make the best use of fluoride ions for dissolving cellulose, the $\mathrm{H}_{2} \mathrm{O} / \mathrm{F}^{-}$ratio should be kept below 2:1.

Acknowledgment. The authors gratefully acknowledge financial support from Södra Cell AB and from the Knowledge Foundation through the graduate school, YPK. Daniel Wiktelius at Göteborg University and Patrik Jarvoll at Chalmers University of Technology are acknowledged for technical support. The Swedish NMR Centre at Gothenburg University is acknowledged for granting spectrometer time and equipment. Istvan Furó at the Royal Institute of Technology (KTH) in Stockholm is gratefully acknowledged for guidance concerning the ${ }^{19} \mathrm{~F}$ NMR interpretation. Several people in Avancell are thanked for valuable discussions.

Supporting Information Available. Three ${ }^{19} \mathrm{~F}$ NMR spectra are herein included, showing the pure solvent TBAF/DMSO, the solvent TBAF/DMSO with $1.3 \%$ added water, and the solvent TBAF/DMSO with $1 \%$ dissolved cellulose, from the bottom to the top. The assigned signals are marked with green vertical lines. This material is available free of charge via the Internet at http://pubs.acs.org.

\section{References and Notes}

(1) Cousins, S. K.; Brown, R. M. Polymer 1995, 36, 3885-3888.

(2) Schulz, L.; Seger, B.; Burchard, W. Biomol. Chem. Phys. 2000, 201, 2008-2022.

(3) Swatloski, R. P.; Spear, S. K.; Holbrey, J. D.; Rogers, R. D. J. Am. Chem. Soc. 2002, 124, 4974-4975.

(4) Dawsey, T. R.; McCormick, C. L. J. Macromol. Sci., Rev. Macromol. Chem. Phys. 1990, C30, 405-440.

(5) Heinze, T.; Dicke, R.; Koschella, A.; Kull, A. H.; Klohr, E.-A.; Koch, W. Macromol. Chem. Phys. 2000, 201, 627-631.

(6) Lu, F.; Ralph, J. Plant J. 2003, 35, 535-544.

(7) Ass, B. A. P.; Frollini, E.; Heinze, T. Macromol. Biosci. 2004, 4, $1008-$ 1013.

(8) Ramos, L. A.; Frollini, E.; Heinze, T. Carbohydr. Polym. 2005, 60, 259-267.

(9) Ramos, L. A.; Frollini, E.; Koschella, A.; Heinze, T. Cellulose 2005, 12, 607-619.
(10) Yeh, A.; Scott, N.; Taube, H. Inorg. Chem. 1982, 21, 2542-2545.

(11) Philipp, B.; Schleicher, H.; Wagenknecht, W. Faserforsch. Textiltech. 1973, 24, 106-112.

(12) Schleicher, H. Acta Polym. 1983, 34, 63-64.

(13) Potthast, A.; Rosenau, T.; Buchner, R.; Röder, T.; Ebner, G.; Bruglachner, H.; Sixta, H.; Kosma, P. Cellulose 2002, 9, 41.

(14) Ishii, D.; Tatsumi, D.; Matsumoto, T.; Murata, K.; Hayashi, H.; Yoshitani, H. Macromol. Biosci. 2006, 6, 293-300.

(15) El-Kafrawy, A. J. Appl. Polym. Sci. 1982, 27, 2435-2443.

(16) Moulthrop, J. S.; Swatloski, R. P.; Moyna, G.; Rogers, R. D. J. Chem. Soc., Chem. Commun. 2005, 12, 1557-1559.

(17) Brendler, E.; Fischer, S.; Leipner, H. Cellulose 2001, 8, 283-288.

(18) Abraham, R. J.; Wileman, D. F.; Bedford, G. R. J. Chem. Soc., Perkin Trans. 2 1973, 1027-1035.

(19) Bodor, A.; Toth, I.; Banyai, I.; Szabo, Z.; Hefter, G. T. Inorg. Chem. 2000, 39, 2530-2537.

(20) Furó, I.; Iliopoulos, I.; Stilbs, P. J. Phys. Chem. B 2000, 104, 485494.

(21) Hindermann, D. K.; Cornwell, C. D. J. Chem. Phys. 1968, 48, 20172025.

(22) Hudlicky, M. J. Fluorine Chem. 1985, 28, 461-472.

(23) According to FMC Biopolymer information centre in 2007.

(24) Fidale, L. C.; Kohler, S.; Prechtl, M. H. G.; Heinze, T.; El Seoud, O. A. Cellulose 2006, 13, 581-592.

(25) Sharma, R. K.; Fry, J. L. J. Org. Chem. 1983, 48, 2112-2114.

(26) Harmon, K. M.; Southworth, B. A.; Harmon, J. J. Mol. Struct. 1993, 300, 339-349.

(27) Berger, S.; Braun, S.; Kalinowski, H.-O. NMR Spectroscopy of the Non-Metallic Elements; John Wiley \& Sons: Chichester, 1997; p 400.

(28) Christe, K. O.; Wilson, W. W. J. Fluorine Chem. 1990, 46, 339-342.

(29) Christe, K. O.; Wilson, W. W. J. Fluorine Chem. 1990, 47, 117-120.

(30) Gerken, M.; Boatz, J. A.; Kornath, A.; Haiges, R.; Schneider, S.; Schroer, T.; Christe, K. O. J. Fluorine Chem. 2002, 116, 49-58.

(31) Christe, K. O.; Wilson, W. W.; Wilson, R. D.; Bau, R.; Feng, J. A. J. Am. Chem. Soc. 1990, 112, 7619-7625.

(32) Tanczos, I.; Borsa, J.; Sajo, I.; Laszlo, K.; Juhasz, Z. A.; Toth, T. Macromol. Chem. Phys. 2000, 201, 2550-2556.

(33) Erdmenger, T.; Haensch, C.; Hoogenboom, R.; Schubert, U. S. Macromol. Biosci. 2007, 7, 440-445.

(34) Hefter, G. T. J. Mol. Liq. 1997, 73/74, 267-276.

(35) Köhler, S.; Heinze, T. Macromol. Biosci. 2007, 7, 307-314.

(36) Hattori, M.; Shimaya, Y.; Saito, M. Polym. J. 1998, 30, 49-55.

(37) Novoselov, N.; Sashina, E.; Petrenko, V.; Zaborsky, M. Fibre Chem. 2007, 39, 153-158.

(38) Xiao, M.; Frey, M. Cellulose 2007, 14, 225-234.

(39) Baldwin, R. L. Biophys. J. 1996, 71, 2056-2063.

BM900667Q 